\title{
A Combination of the Korean Version of the Mini-Mental State Examination and Korean Dementia Screening Questionnaire Is a Good Screening Tool for Dementia in the Elderly
}

\author{
Mi Hee Shin ${ }^{1}$, Young Min Lee ${ }^{1,2} \bowtie$, Je Min Park ${ }^{1,2}$, Cheol Joong Kang ${ }^{1,2}$, \\ Byung Dae Lee ${ }^{1,2}$, Eunsoo Moon ${ }^{1,2}$ and Young In Chung ${ }^{2,3}$ \\ ${ }^{1}$ Department of Psychiatry, Pusan National University Hospital, Busan, Korea \\ ${ }^{2}$ Department of Psychiatry, School of Medicine, Pusan National University, Busan, Korea \\ ${ }^{3}$ Department of Psychiatry, Pusan National University Yangsan Hospital, Yangsan, Korea
}

Objective The aim of this study is to investigate whether a combination of the Korean version of the mini-mental state examination (K-MMSE) and the Korean dementia screening questionnaire (KDSQ) is better than the use of test alone when differentiating patients with dementia from those without dementia in Korea.

Methods The subjects (patients without dementia, 1120; patients with dementia, 908) were recruited from the Clinical Research Center for Dementia of South Korea. K-MMSE and KDSQ were used. Diagnosis of dementia was made according to the Diagnostic and Statistical Manual of Mental Disorders, fourth Edition. The weighted sum rule derived from logistic regression analysis was used for the combination of K-MMSE and KDSQ

Results On comparing the Area Under the Curve for each test using the method of Hanley and McNeil, the weighted sum was significantly greater than KDSQ or K-MMSE, and K-MMSE was significantly greater than KDSQ.

Conclusion This study shows that when differentiating patients with dementia from those without dementia in Korea, a combination of K-MMSE and KDSQ achieved using the weighted sum method is better than either test performed alone. Further epidemiological studies in community-based settings are required before our results can be generalized to nonclinical samples.

Psychiatry Investig 2011;8:348-353

Key Words Dementia, screening, Korean version of the Mini-Mental State Examination,

Korean Dementia Screening Questionnaire.

\section{INTRODUCTION}

It is an accepted fact that dementia is a clinical and public health concern. It was estimated that in 2005, 24.3 million people worldwide had dementia, and this number is estimated to rise to 81.1 million by $2040 .{ }^{1}$ In Korea, there are presently about 400,000 people with dementia, and this number is expected to rise to 800,000 million in just 20 years. ${ }^{2}$ However, it is currently known that only a third to a half the people with

Received: June 3, 2011 Revised: September 1, 2011

Accepted: September 2, 2011 Available online: December 8, 2011

$\triangle$ Correspondence: Young Min Lee, MD

Department of Psychiatry, School of Medicine, Pusan National University, 305 Gudeok-ro, Seo-gu, Busan 602-739, Korea

Tel: +82-51-240-7301, Fax: +82-51-248-3648, E-mail: psyleekr@naver.com

(c) This is an Open Access article distributed under the terms of the Creative Commons Attribution Non-Commercial License (http://creativecommons.org/licenses/bync/3.0) which permits unrestricted non-commercial use, distribution, and reproduction in any medium, provided the original work is properly cited. dementia receive a formal diagnosis or contact specialist services. ${ }^{3}$ Such diagnosis or contact is often made only well into the late stage of illness when the possibility of prevention or intervention is limited. Early diagnosis and intervention can, in many cases, help delay institutionalization, and also give patients and their families time to plan their future. ${ }^{3}$

A screening method is important for the early diagnosis of dementia. Direct brief testing of the patient's cognition by a trained rater using an established test is the most common form of screening for dementia, but informant reports are an alternate method of detecting dementia. ${ }^{4}$ In Korea, a widely used approach for screening dementia is the conduction of the Korean versions of the mini-mental state examination (KMMSE; brief cognitive tests), ${ }^{5,6}$ which provide a summary of global cognitive functions, and the use of the Korean dementia screening questionnaire (KDSQ; informant reports), ${ }^{7}$ 
which provides information about changes in an elderly patient's cognitive performance.

There are advantages and disadvantages to brief cognitive tests and informant reports. Brief cognitive tests can be influenced by education and pre-morbid ability ${ }^{8}$ and require subjects to have intact language, sensory, and motor abilities. ${ }^{9}$ Informant reports are unaffected by a subject's education or premorbid intelligence, ${ }^{10}$ but performance may be influenced by both the personality and the affective state of the subject and the informant and the nature of their relationship. ${ }^{11}$

It is likely that the supplementation of brief cognitive tests with informant reports may improve accuracy when screening for dementia in clinical settings. ${ }^{4,12-16}$ However, previous studies examining the combination of brief cognitive tests and informant reports have shown conflicting results. The results of some studies have suggested improvement in screening, ${ }^{4,16}$ but those of another study have been equivocal, ${ }^{13}$ wherein a combination of brief cognitive tests and informant reports in the elderly was compared with the individual use of brief cognitive tests or informant reports. Therefore, there is no general agreement on the view that a combination of brief cognitive tests and informant reports improves detection of dementia. The aim of this study is to investigate whether a combination of K-MMSE and KDSQ is better than either one test alone in distinguishing patients with dementia from those without dementia in Korea.

\section{METHODS}

\section{Study subjects}

The subjects were recruited from the Clinical Research Center for Dementia of South Korea (CREDOS). CREDOS is a 9-year longitudinal study whose patients have been followed up every 12 months after enrollment; this study is funded by the Korean Ministry of Health, Welfare and Family Affairs (http://www.crcd.or.kr). Fifty-six hospitals have participated in this study, yielding a nationwide hospital-based study cohort. The 2028 subjects who visited the hospital were included in the study; there were 908 patients with dementia and 1120 patients without dementia. Patients were excluded if they had no informant or had a serious medical condition, severe sensorimotor deficit, or depressive symptoms (geriatric depression scale; GDS >15). ${ }^{17}$ Written informed consent was obtained from the participants in this study, and this study was approved by the Pusan National University Hospital Institutional Review Board.

\section{Dementia screening instruments}

$\mathrm{K}-\mathrm{MMSE}$ and the KDSQ were used. The MMSE is a brief test of mental status and has been validated for use in many countries. ${ }^{5}$ The K-MMSE used in this study was a version that has been previously modified to some extent for use in Korea and has been shown to exhibit properties similar to those of the original MMSE. ${ }^{6} \mathrm{KDSQ}$ developed in Korea is a self-administered questionnaire that asks an informant about changes in an elderly patient's cognitive performance over the past 1 year. ${ }^{7} \mathrm{KDSQ}$ has 15 items on cognitive dysfunction, each rated on a 3-point scale from 0 (no change) to 2 (frequent change), with 1 representing occasional change. KDSQ is not influenced by age or level of education. This instrument has shown high validity and reliability in distinguishing between patients with dementia and those without dementia in Korea. ${ }^{\text {? }}$

\section{Diagnosis of dementia}

Diagnoses of dementia was made according to the Diagnostic and Statistical Manual of Mental Disorders, fourth Edition criteria ${ }^{18}$ by psychiatrists or neurologists who used all the available information, except the scores of screening instruments, and diagnosis was reviewed by a committee consisting of 2 psychiatrists and 2 neurologists before the patient sample was enrolled in CREDOS. Especially, Alzheimer's disease $(\mathrm{AD})$ was diagnosed according to the criteria of the National Institute of Neurological and Communicative Disorders and Stroke and the Alzheimer's Disease and Related Disorders Association. ${ }^{24}$ Vascular dementia (VaD) was diagnosed according to the criteria of the National Institute of Neurological Disorders and Stroke-Association Internationale pour la Recherche et l'Enseignement en Neurosciences. ${ }^{25}$

The sample of patients without dementia included individuals with diagnoses of mild cognitive impairment $(\mathrm{MCI})^{19,20}$ or subjective memory impairment (SMI) ${ }^{21}$ and healthy subjects who did not have any cognitive impairment.

\section{Combination of K-MMSE and KDSQ: using weighted sum rule}

We combined the scores obtained for K-MMSE and KDSQ using the weighted sum rule suggested by Mackinnon and Mulligan. ${ }^{4}$ In the weighted sum rule, the 2 test scores are combined to produce a score on a new scale before a cut point is calculated that best differentiates cases from non-cases. The formula for the weighted sum rule was derived from logistic regression analysis, and Receiver operating characteristics (ROC) curves were constructed. The weighted sum rule allowed a graduated trade-off between the scales. If a patient is judged not impaired on one test, greater evidence to suggest impairment is required on the other test for it to qualify as a case.

\section{Statistical analysis}

Demographic and clinical variables were compared using 
Chi-square tests for categorical variables and the Mann-Whitney $U$ test for variables that were not normally distributed. Logistic regression analysis was used to assess whether a relationship exists between caseness and one or more predictor variables, and to derive the optimal equation for predicting the probability of caseness. ROC analysis was used to assess the effectiveness of each test or combination of test as a screening method for dementia. The area under the curve (AUC) for each test was compared using the method of Hanley and McNeil (MedCalc version 11.4). ${ }^{22}$ The analysis, excluding the comparison of AUC, was performed using SPSS for Windows, version 15.0 (SPSS Inc., Chicago, IL). Statistical tests were 2-tailed, and results were regarded as significant at or below the $5 \%$ probability level.

\section{RESULTS}

\section{Demographic and clinical data}

Table 1 shows the distribution of the demographic characteristics, GDS, and KDSQ and K-MMSE scores of the patient sample. Patients with dementia were 5 years older than those without dementia ( $74.55 \pm 7.39$ vs. $69.64 \pm 8.04 ; Z=-13.93 ; \mathrm{p}<$ 0.001 ). The level of education was less by 1.5 years in patients with dementia than in those without dementia (7.24 \pm 5.35 vs. 8.61 $\pm 5.34 ; Z=-5.82 ; \mathrm{p}<0.001)$. Patients with dementia were more impaired than those without dementia as reflected by their KDSQ scores $(13.71 \pm 6.68$ vs. $5.95 \pm 4.29 ; Z=-26.40 ; \mathrm{p}<$ $0.001)$ and K-MMSE scores (19.14 \pm 5.03 vs. $25.71 \pm 3.48 ; \mathrm{Z}=$ -28.19; $\mathrm{p}<0.001)$. The patient groups did not show a significant difference in terms of gender $\left(\mathrm{x}^{2}=1.71 ; \mathrm{df}=1 ; \mathrm{p}=0.19\right)$ and GDS (8.36 4.17 vs. $8.71 \pm 4.05 ; Z=-1.77 ; p=0.076)$.

Table 2 shows the composition of diagnosis and severity in the patient sample. The MCI subjects were 3 times as many as SMI or healthy subjects ( $73.3 \%$ vs. $26.7 \%$ ) in the sample of patients without dementia. The AD subjects were 6 times as many as $\mathrm{VaD}$ subjects ( $85.7 \%$ vs. $14.3 \%$ ) in the sample of patients with dementia. Clinical dementia rating (CDR) 0.5 (88.8 $\%$ ) has the highest frequency in the sample of patients without dementia. In the sample of patients with dementia, CDR 1 (49.6\%) has the highest frequency, and CDR 0.5 (37.4\%), the next highest frequency.

\section{Logistic regression analysis}

The logistic regression analysis was performed to obtain the weighted sum equation used to predict dementia from the scores obtained for KDSQ and K-MMSE. The weighted sum rule of combining the KDSQ and K-MMSE scores is derived from the logistic regression analysis shown in the following equation.

Logit $($ case $)=4.644+0.204 \times$ KDSQ $-0.293 \times$ K-MMSE

$$
\text { or } \operatorname{Pr}(\text { case })=\frac{1}{1+\mathrm{e}^{-(4.644+0.204 \times \text { KDSQ- }-0.293 \times \mathrm{K}-\mathrm{MMSE})}}
$$

Where logit (case) is the logarithm of the odds of a subject having dementia, which is equal to log (probability of a case/ probability of non-case). $\operatorname{Pr}$ (case) is the probability of a case of dementia. KDSQ and MMSE are the test score values.

\section{Receiver operating characteristic analysis}

Sensitivity, specificity, and the AUC for the KDSQ and KMMSE scores and the weighted sum rule for distinguishing between patients with dementia and those without dementia are shown in Table 3. Figure 1 shows the results of the ROC analysis for the weighted sum method and KDSQ and K-

Table 1. Demographic characteristics of the patient sample

\begin{tabular}{|c|c|c|}
\hline $\begin{array}{l}\text { Demographic } \\
\text { characteristics }\end{array}$ & $\begin{array}{l}\text { No dementia } \\
(\mathrm{N}=1,120)\end{array}$ & $\begin{array}{c}\text { Dementia } \\
(\mathrm{N}=908)\end{array}$ \\
\hline Age, mean $\pm \mathrm{SD}$, years ${ }^{\dagger}$ & $69.64 \pm 8.04$ & $74.55 \pm 7.39$ \\
\hline \multicolumn{3}{|l|}{ Gender* } \\
\hline Male, n (\%) & $415(37.1)$ & $311(34.3)$ \\
\hline Female, n (\%) & $705(62.9)$ & $597(65.7)$ \\
\hline Education, years $^{\dagger}$ & $8.61 \pm 5.34$ & $7.24 \pm 5.35$ \\
\hline GDS & $8.71 \pm 4.05$ & $8.36 \pm 4.17$ \\
\hline $\mathrm{KDSQ}^{\dagger}$ & $5.95 \pm 4.29$ & $13.71 \pm 6.68$ \\
\hline $\mathrm{K}_{-\mathrm{MMSE}^{\dagger}}$ & $25.71 \pm 3.48$ & $19.14 \pm 5.03$ \\
\hline
\end{tabular}

p value $<0.001 .{ }^{*}$ Chi-square test, ${ }^{\dagger}$ Mann-Whitney $U$ test. GDS: Geriatric Depression Scale, n: number, SD: standard deviation, KDSQ: Korean Dementia Screening Questionnaire, K-MMSE: Korean version of the Mini-Mental State Examination

Table 2. Composition and severity of the patient sample

\begin{tabular}{lcc}
\hline & & No dementia $(\mathrm{N}=1,120)$ \\
\hline Diagnosis & SMI or healthy, n (\%) & $299(26.7)$ \\
& MCI, n (\%) & $821(73.3)$ \\
Severity & CDR 0 & $114(10.2)$ \\
& CDR 0.5 & $1006(88.8)$ \\
& CDR (SOB), mean \pm SD & $1.25 \pm 0.92$ \\
\hline Diagnosis & AD, n (\%) & Dementia (N=908) \\
& VaD, n (\%) & $130(14.3)$ \\
Severity & CDR 0.5, n (\%) & $340(37.4)$ \\
& CDR 1, n (\%) & $450(49.6)$ \\
& CDR 2, n (\%) & $109(12.0)$ \\
& CDR 3, n (\%) & $9(1)$ \\
\hline
\end{tabular}

$\mathrm{AD}$ : alzheimer's disease, CDR: clinical dementia rating, $\mathrm{CDR}$ (SOB): clinical dementia rating (sum of box scores), MCI: mild cognitive impairment, n: number, SD: standard deviation, SMI: subjective memory impairment, $\mathrm{VaD}$ : vascular dementia 
Table 3. Individual test (KDSQ, K-MMSE) and combination test (Weighted sum) in dementia screening

\begin{tabular}{lcccc}
\multicolumn{1}{c}{ Test } & Sensitivity & Specificity & AUC & 95\% CI \\
\hline KDSQ (8/9 cut-point) & $75.2 \%$ & $73.2 \%$ & 0.840 & $0.823-0.857$ \\
K-MMSE (24/25 cut-point) & $86.0 \%$ & $71.2 \%$ & 0.863 & $0.847-0.879$ \\
Weighted sum [logit (case) $\geq-0.932]$ & $89.2 \%$ & $73.4 \%$ & 0.906 & $0.893-0.919$ \\
\hline
\end{tabular}

AUC: area under the curve, CI: confidence interval, KDSQ: Korean Dementia Screening Questionnaire, K-MMSE: Korean version of the Mini-Mental State Examination

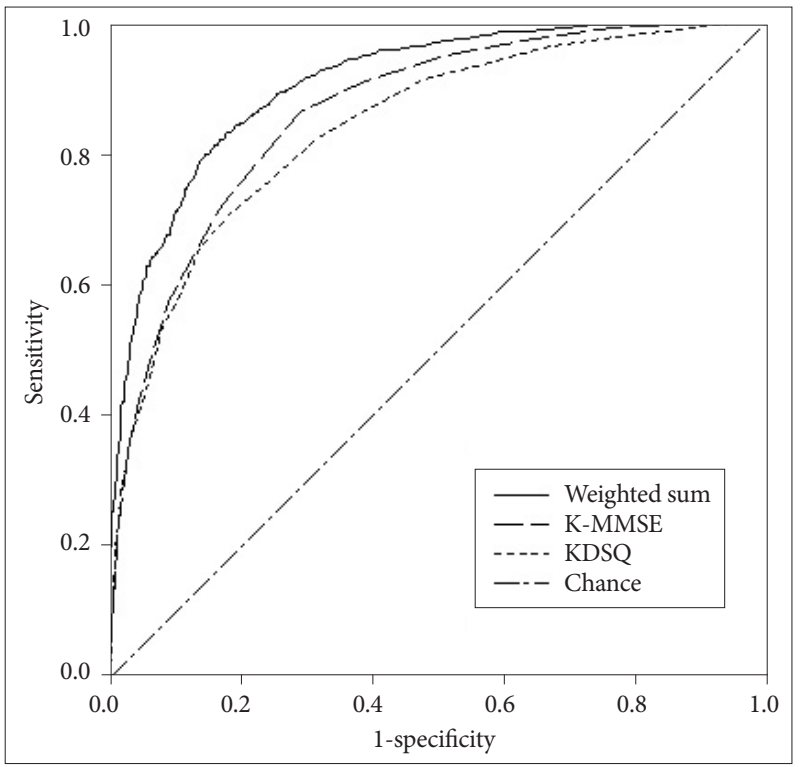

Figure 1. ROC curve. Comparisons between the weighted sum method, K-MMSE, and the KDSQ in screen for dementia. KDSQ: Korean Dementia Screening Questionnaire, K-MMSE: Korean version of the Mini-Mental State Examination, ROC: Receiver operating characteristics.

MMSE for distinguishing between patients with dementia $(\mathrm{n}=908)$ and those without dementia $(\mathrm{n}=1,120)$. By using the method of Hanley and McNeil (MedCalc version 11.4), ${ }^{22}$ it was found that the AUC for the weighted sum rule was significantly greater than that for KDSQ (0.906 vs. $0.840 ; Z=9.78$; $\mathrm{p}<0.001$ ) and K-MMSE (0.906 vs. $0.863 ; \mathrm{Z}=7.88 ; \mathrm{p}<0.001$ ), and there was significant difference between the AUCs for KMMSE and KDSQ ( 0.863 vs. $0.840 ; \mathrm{Z}=2.107 ; \mathrm{p}=0.035$ ). Thus, in our study, the weighted sum rule has an advantage over KMMSE or KDSQ scores in screening for dementia.

\section{DISCUSSION}

Our results demonstrate that a combination of brief cognitive testing and the informant report was better than either test used alone in the screening for dementia among patients visiting a hospital. This study shows that a combination of KMMSE and KDSQ achieved using the weighted sum method is better than either test used alone in distinguishing between patients with dementia and those without dementia in Korea. On comparing the AUC for each test using the method of
Hanley and McNeil, ${ }^{22}$ we found that the weighted sum was significantly greater than KDSQ and K-MMSE (Figure 1). This is consistent with results of previous studies., ${ }^{4,14,16}$ Mackinnon and Mulligan ${ }^{4,14}$ showed that a combination of MMSE and informant report achieved using the weighted sum method was better than either test used alone. ${ }^{14}$ Narasimhalu et al. ${ }^{16}$ recommended that the informant report be used along with MMSE whenever available and that the weighted sum method be used to combine the MMSE and informant report, particularly in populations with a low level of education. Brief cognitive tests and informant reports in screening for dementia do not always measure the same cognitive domains of a subject. ${ }^{4}$ Correlations between the 2 types of screening methods are moderate. ${ }^{11}$ Moreover, Jorm et al..$^{23}$ in their analyses of brief cognitive test and informant report items found that there are separate factors in each item domain. The 2 types of screening methods measure rather different things. Therefore, the supplementation of brief cognitive tests with informant reports may improve accuracy when screening for dementia in our study.

In the present study, K-MMSE outperformed KDSQ. On comparing the AUC for each test using the method of Hanley and McNeil, we found that K-MMSE was significantly greater than KDSQ (Figure 1). Previous studies on western populations that have examined the combination of the brief cognitive tests and informant reports have reported significantly better AUC results using the MMSE than when using informant reports. ${ }^{4,13,14}$ However, in another study of the populations in a developing country, ${ }^{16}$ the informant reports always outperformed MMSE. There are several possible reasons why different results are produced by MMSE and informant reports: First, the difference between educational levels may be one of the reasons. The level of education of populations in developing countries is lower than that of populations in western countries. ${ }^{4,13,14,16}$ Informant reports are unaffected by a subject's education and pre-morbid intelligence; hence, these will be beneficial when screening for dementia in patients with a low level of education, because MMSE is affected by an education-related bias. Second, the presence or absence of a reliable informant may be another reason. In western countries, the elderly often live independently. Therefore, informant reports may be less accurate in western countries than 
in developing countries. Third, differences in the compositions of the patient sample may be another reason. A previous study in which informant reports were shown to outperform MMSE included patients participating in a study of cognition after stroke. ${ }^{16}$ Hence, the frequency of $\mathrm{VaD}$ in their study was higher than that of $\mathrm{VaD}$ in our study (48.5\% vs. $14.3 \%$ ). They reported that the informant reports produced better AUCs for differentiating patients with $\mathrm{VaD}$ from those without dementia than for differentiating patients with dementia from those without dementia ( 0.93 vs. 0.89 ). Hence, the higher frequency of $\mathrm{VaD}$ in a patient sample may be associated with better AUC results from informant reports.

The results for sensitivity and specificity of KDSQ, KMMSE, and the weighted sum rule were similar to those of other studies., ${ }^{4,1416}$ We found that the sensitivity of K-MMSE with a 24/25 cut point was higher than that reported by Mackinnon and Mulligan $^{4}$ (0.86 vs. 0.76$)$; however, the specificity was lower in our study ( 0.73 vs. 0.90 ). For KDSQ (8/9 cut point), we found that sensitivity and specificity were slightly lower than those reported by Yang et al. $:$ : they reported a sensitivity of 0.79 and a specificity of 0.80 , whereas we reported 0.75 sensitivity and 0.73 specificity. The weighted sum rule (logit (case) $\geq-0.932$ ) produced a better result in the study by Mackinnon and Mulligan ${ }^{4}$ than in the present study: the former found a sensitivity of 0.93 and a specificity of 0.85 , whereas we reported a sensitivity of 0.89 and specificity of 0.73 . Although the results for sensitivity and specificity were similar to those in other studies, ${ }^{4,7,14,16}$ the specificity was somewhat low in our study.

One possible reason for the lower specificity values in our study is differences in dementia severities among the patient sample. The MCI subjects were three times as many as SMI or healthy subjects $(73.3 \%$ vs. $26.7 \%)$ in no dementia sample. Hence, the fewer number of normal individuals could lead to increase difficulty in differentiating patients with patients from those without dementia using the tests. Further research is needed to establish the effect of the difference in dementia severities among the patient sample on screening for dementia. In our study, the weighted sum rule had a higher sensitivity and specificity than K-MMSE and KDSQ (Table 3). This is consistent with the results of previous studies. ${ }^{4,14,16}$

The present study has several limitations. First, the main disadvantage is that the study samples were selected from a clinical population. Informant reports collected from subjects in a clinical setting may be more reliable than those collected from subjects in community-based settings. On the other hand, MMSE, which can be utilized without informant input, can be more appropriate in community-based studies. Hence, our finding should not be generalized to nonclinical samples. Second, the informant reports may be influenced by the qual- ity of the relationship between the patient and informant. If there are no reliable informants, the tests are inapplicable to patients. Third, because it is a multicenter study, there are many examiners. In conclusion, this study shows a combination of K-MMSE and KDSQ is better than either test used alone when differentiating patients with dementia from those without dementia in Korea. Further epidemiological studies in community-based settings are required before our results can be generalized to nonclinical samples.

\section{Acknowledgments}

This study was supported by a grant of the Korea Healthcare technology R\&D Project, Ministry of Health and Welfare, Republic of Korea. (A102065) and of Pusan National University Hospital.

\section{REFERENCES}

1. Ferri CP, Prince M, Brayne C, Brodaty H, Fratiglioni L, Ganguli M, et al. Global prevalence of dementia: a Delphi consensus study. Lancet 2005;366:2112-2117.

2. Kim KW, Park JH, Kim MH, Kim MD, Kim BJ, Kim SK, et al. A nationwide survey on the prevalence of dementia and mild cognitive impairment in South Korea. J Alzheimers Dis 2011;23:281-291.

3. Overcoming barriers to early dementia diagnosis. Lancet $2008 ; 371$ : 1888.

4. Mackinnon A, Mulligan R. Combining cognitive testing and informant report to increase accuracy in screening for dementia. Am J Psychiatry 1998;155:1529-1535.

5. Folstein MF, Folstein SE, McHugh PR. "Mini-mental state". A practical method for grading the cognitive state of patients for the clinician. J Psychiatr Res 1975;12:189-198.

6. Han C, Jo SA, Jo I, Kim E, Park MH, Kang Y. An adaptation of the Korean mini-mental state examination (K-MMSE) in elderly Koreans: demographic influence and population-based norms (the AGE study). Arch Gerontol Geriatr 2008;47:302-310.

7. Yang DW, Cho BL, Chey JY, Kim SY, Kim BS. The Development and Validation of Korean Dementia Screening Questionnaire (KDSQ). J Korean Neurol Assoc 2002;20:135-141.

8. O'Connor DW, Pollitt PA, Hyde JB, Fellows JL, Miller ND, Brook CP, et al. The reliability and validity of the Mini-Mental State in a British community survey. J Psychiatr Res 1989;23:87-96.

9. Tombaugh TN, McIntyre NJ. The mini-mental state examination: a comprehensive review. J Am Geriatr Soc 1992;40:922-935.

10. Jorm AF, Jacomb PA. The Informant Questionnaire on Cognitive Decline in the Elderly (IQCODE): socio-demographic correlates, reliability, validity and some norms. Psychol Med 1989;19:1015-1022.

11. Jorm AF. The Informant Questionnaire on cognitive decline in the elderly (IQCODE): a review. Int Psychogeriatr 2004;16:275-293.

12. Jorm AF. The value of informant reports for assessment and prediction of dementia. J Am Geriatr Soc 2003;51:881-882.

13. Knafelc R, Lo Giudice D, Harrigan S, Cook R, Flicker L, Mackinnon A, et al. The combination of cognitive testing and an informant questionnaire in screening for dementia. Age Ageing 2003;32:541-547.

14. Mackinnon A, Khalilian A, Jorm AF, Korten AE, Christensen H, Mulligan R. Improving screening accuracy for dementia in a community sample by augmenting cognitive testing with informant report. J Clin Epidemiol 2003;56:358-366.

15. Senanarong V, Assavisaraporn S, Sivasiriyanonds N, Printarakul T, Jamjumrus P, Udompunthuruk S, et al. The IQCODE: an alternative screening test for dementia for low educated Thai elderly. J Med Assoc Thai 2001;84:648-655.

16. Narasimhalu K, Lee J, Auchus AP, Chen CP. Improving detection of de- 
mentia in Asian patients with low education: combining the MiniMental State Examination and the Informant Questionnaire on Cognitive Decline in the Elderly. Dement Geriatr Cogn Disord 2008;25:17-22.

17. Yesavage JA, Brink TL, Rose TL, Lum O, Huang V, Adey M, et al. Development and validation of a geriatric depression screening scale: a preliminary report. J Psychiatr Res 1982-1983;17:37-49.

18. American Psychiatric Association. Diagnostic and Statistical Manual of Mental Disorders, 4th Edition. Washington, DC: American Psychiatric Press; 1994.

19. Petersen RC, Smith GE, Waring SC, Ivnik RJ, Tangalos EG, Kokmen E. Mild cognitive impairment: clinical characterization and outcome. Arch Neurol 1999;56:303-308.

20. Graham JE, Rockwood K, Beattie BL, Eastwood R, Gauthier S, Tuokko $\mathrm{H}$, et al. Prevalence and severity of cognitive impairment with and without dementia in an elderly population. Lancet 1997;349:1793-1796.

21. Abdulrab K, Heun R. Subjective Memory Impairment. A review of its definitions indicates the need for a comprehensive set of standardised and validated criteria. Eur Psychiatry 2008;23:321-330.

22. Hanley JA, McNeil BJ. The meaning and use of the area under a receiver operating characteristic (ROC) curve. Radiology 1982;143:29-36.

23. Jorm AF, Mackinnon AJ, Henderson AS, Scott R, Christensen H, Korten AE, et al. The Psychogeriatric Assessment Scales: a multi-dimensional alternative to categorical diagnoses of dementia and depression in the elderly. Psychol Med 1995;25:447-460.

24. McKhann G, Drachman D, Folstein M, Katzman R, Price D, Stadlan EM. Clinical diagnosis of Alzheimer's disease: report of the NINCDSADRDA Work Group under the auspices of Department of Health and Human Services Task Force on Alzheimer's Disease. Neurology 1984;34: 939-944.

25. Roman GC, Tatemichi TK, Erkinjuntti T, Cummings JL, Masdeu JC, Garcia JH, et al. Vascular dementia: diagnostic criteria for research studies. Report of the NINDS-AIREN International Workshop. Neurology 1993;43:250-260. 